\title{
Whole genome sequence comparison of ten diagnostic brucellaphages propagated on two Brucella abortus hosts
}

Ekaterine Tevdoradze ${ }^{1 \dagger}$, Jason Farlow ${ }^{3,4^{*} \dagger}$, Adam Kotorashvili ${ }^{2}$, Natia Skhirtladze ${ }^{1}$, Irina Antadze ${ }^{1}$, Sophio Gunia ${ }^{1}$ Nana Balarjishvili', Leila Kvachadze ${ }^{1}$ and Mzia Kutateladze ${ }^{1}$

\begin{abstract}
Background: Recently the genome sequences of two brucellaphages, isolated in Georgia (Tb) and Mexico (Pr) were reported revealing pronounced sequence homogeneity and the presence of two major indels discriminating the two phages. Subsequent genome sequencing of six diagnostic brucellaphages: Tbilisi (Tb), Firenze (Fz), Weybridge (Wb), S708, Berkeley (Bk) and R/C phages identified three major genetic groups. However, the propensity for fine-scale genetic variability of diverse brucellaphages grown on multiple hosts within a single Brucella species remains unknown.
\end{abstract}

Methods: We sequenced the complete genomes of ten brucellaphages following initial propagation on B. abortus strain 141 and after subsequent propagation on B. abortus strain S19. All brucellaphages were isolated and propagated at the Eliava Institute including the original Tb phage. Genomic libraries were quantified using the Qbit and sheared on the Covaris M220. QC for fragmentation was performed on the BioAnalyzer 2100. DNA libraries were prepared using an Illumina Paired-End protocol and sequenced on the Illumina MiSeq. Sequence analysis was performed using Geneious and MEGA software.

Results: Comparative whole genome sequence analysis revealed genetic homogeneity consistent with previously published data as well as multiple nucleotide variations. Genomic changes as a result of passages were observed in similar genes and predominantly occurred at identical sites in separate phages. Multiple instances of within-sample genetic heterogeneity were observed often at shared genomics positions across phages. Positive selection was detected in the tail collar protein gene. We also identified a Staphylothermus marinus F1-like CRISPR spacer and sequences orthologous to both prophage antirepressors of Brucella spp. and intergenic sequences encoded by Ochrobactrum anthropi.

Conclusion: We surveyed whole genome level diversity in phage lytic for B. abortus as they are propagated on alternate vaccine strains within the species. Our data extend previous results indicating select variable hotspots and broad genomic homogeneity as well as multiple common polymorphisms and within-sample variation. These data also provide additional genomes for future reference in comparative studies involving the molecular evolution and host specificity of brucellaphages.

\footnotetext{
* Correspondence: jasonfarlow@hotmail.com

${ }^{\dagger}$ Equal contributors

${ }^{3}$ Academic Engagement Program (AEP) Pennsylvania State University, University Park, State College, USA

${ }^{4}$ Farlow Scientific Consulting Company, LLC, Lewiston, Utah, USA

Full list of author information is available at the end of the article
} 


\section{Introduction}

Brucellosis is an important global zoonotic disease caused by members of the Brucella genus [1]. Members of this genus exhibit distinct primary host specificity and currently comprise ten species: $B$. melitensis, $B$. suis, $B$. abortus, B. ovis, B. canis, B. neotomae, B. ceti, B. microti, B. pinnipedialis, and $B$. inopinata, (http://www.bacterio.net/ brucella.html). Typing of Brucella is confirmed by serum agglutination, colony morphology, $\mathrm{CO}_{2}$ requirement, $\mathrm{H}_{2} \mathrm{~S}$ production, growth on thionine or basic fuchsine plates on serum dextrose agar (SDA), and diagnostic brucellaphage lysis [2,3]. Phage typing of Brucella is an established diagnostic and is used routinely in many diagnostic applications [4-6].

Recently, whole genome sequences of the Tbilisi phage (hereafter designated $\mathrm{Tb}_{M}$ ) and a brucellaphage isolated in Mexico ( $\mathrm{Pr}$ ) were reported that revealed pronounced sequence homogeneity (98.7\%) and the presence of two major indels discriminating the two strains [7]. The brucellaphage genomes also encoded a prokaryotic-like DNA replication factor and a bacterial origin of replication (OriC) [7]. Many of the virion proteins show similarity to a cryptic prophage encoded by Chelativorans and based on amino acid similarity and gene synteny the authors suggest common ancestry between these phages that share evolutionarily related host species (Brucella and Chelativorans). Subsequent comparative whole genome analysis of six diagnostic brucellaphages including Tbilisi (Tb), Firenze (Fz), Weybridge (Wb), S708, Berkeley (Bk) and $\mathrm{R} / \mathrm{C}$ phages revealed high sequence similarity and identified three major genetic groups consistent with their defined host range phenotypes [8]. Group I comprised $\mathrm{Tb}$ and $\mathrm{Fz}$ phages predominantly lytic for B. abortus and $B$. neotomae while Group II included $\mathrm{Bk}, \mathrm{R} / \mathrm{C}$, and Pr phages lytic mainly for $B$. abortus, B. melitensis and $B$. suis. Phages Wb and S708 lytic for B. suis, B. abortus, and $B$. neotomae comprised Group III. The Tb phage sequenced by Flores et al was obtained from the Gamaleya Institute of Epidemiology and Microbiology, Moscow, Russia. This genome displayed comparatively greater genetic distance from all other within-group brucellaphages including an alternate $\mathrm{Tb}$ phage (designated $\mathrm{Tb}_{W}$ ) [7] obtained from the Félix d'Hérelle Reference Center in Canada. The two major indels matching those initially observed in the $\mathrm{Tb}_{M}$ and the Pr phage genomes [7] comprised the major discriminating genetic feature distinguishing major evolutionary groups of brucellaphages [8]. The distinct genetic divergence of the $\mathrm{Tb}_{M}$ genome sequence was suggested to have resulted from diversifying selection and/or genetic drift in the laboratory. The study also showed the putative phage collar protein is a hotspot for genetic variation across the major evolutionary groups and is currently undergoing positive Darwinian selection [8]. The three major evolutionary clades that comprise the evolutionary structure of brucellaphages may in-part reflect particular adaptations to differences in unique host attributes [8]. The propensity for fine-scale genetic variability of brucellaphages grown within individual Brucella spp. remains unknown. Such data may elucidate further the fine-scale genetic structure of this phage population and may facilitate discovery of biological determinants mediating within-species variations in host range.

In order to assess the evolutionary structure and genomic plasticity of brucellaphages following propagation on alternate host strains within the $B$. abortus species we sequenced the complete genomes of ten brucellaphages following propagation first on B. abortus strain 141 and after subsequent propagation on host strain S19. Phages included in this study comprised a set of ten brucellaphages lytic for $B$. abortus recently characterized at the Eliava Institute during efforts to expand the diagnostic repertoire of phages currently used in the country of Georgia. Our data revealed patterns in the genomic diversity of these phages including potential neutral and/or adaptive nucleotide polymorphisms within a set of genes that shared common sequence mutations.

\section{Materials and methods \\ Brucellaphages and bacterial strains Phage specificity and purification}

All brucellaphages analyzed were originally isolated at Eliava including the original $\mathrm{Tb}$ phage now used worldwide (isolated from manure), 1066 (isolated from B. canis), 281 (B. ovis), 02 (B. ovis), 177 (B. melitensis), 110 (B. melitensis), V (B. abortus), 11sa (B. suis), 544 (B. abortus), 141 (B. abortus 141) (Table 1 ). Highly concentrated phages were obtained on Petri dishes (plate concentrate); purification of native virions was carried out by gradient centrifugation at $18,000 \mathrm{G}$ at $4^{\circ} \mathrm{C}$. Briefly, Phage lysates were centrifuged at $5000 \mathrm{~g}, 4 \mathrm{C}$ to remove bacterial debris, then the suspension was centrifuged at 18000 g. Phage were suspended in SSC buffer $(0.15 \mathrm{mM}$ $\mathrm{NaCl}, 0.015 \mathrm{mM} \mathrm{Na}$ citrate, $\mathrm{pH}$ 7.0). Final purification of native virions was carried out by centrifugation at $18000 \mathrm{~g}$ through a $\mathrm{CsCl}$ gradient in an SW25 rotor using a Beckman L2-65B ultracentrifuge at $4^{\circ} \mathrm{C}$. Phage fractions were dialysed against SSC. Single-step growth studies were performed as described previously (Adams, 1961, p. 416). Phage were independently propagated to limit contamination on freshly prepared bacterial lawns; plaque number was determined after $24 \mathrm{~h}$ and $48 \mathrm{~h}$ of incubation at $37^{\circ} \mathrm{C}$ then plaque forming units (pfu) per $\mathrm{ml}$ were calculated. Biological properties such as adsorption, latent period, lysis time and average burst size were studied by standard methodology [9]. 
Table 1 Reproduction parameters of phages used in this study

\begin{tabular}{|c|c|c|c|c|c|c|}
\hline Phages & Isolation source & Time of adsorption (min) & $\%$ adsorption & Latent period (min) & Lysis time (min) & Average burst size (PFU/ml) \\
\hline Tb_141 & liquid manure & 190 & 90 & 200 & 460 & $40-46$ \\
\hline Tb_19 & liquid manure & 180 & 81 & 210 & 460 & $50-53$ \\
\hline 110 & B. melitensis & 120 & 70 & $180-220$ & 440 & $18-20$ \\
\hline 141 & B. abortus & 120 & 76 & 240 & 450 & $27-30$ \\
\hline $11 \mathrm{sa}$ & B. suis & 120 & 53 & $210-240$ & 410 & $28-30$ \\
\hline 1066 & B. canis & 150 & 98 & 180 & 425 & $110-120$ \\
\hline 102 & B. ovis & 120 & 82 & 240 & 400 & $36-40$ \\
\hline 544 & B. abortus & $90-100$ & 82 & $270-300$ & $480-490$ & $30-35$ \\
\hline 281 & B. ovis & $90-100$ & 70 & $250-280$ & $450-460$ & $60-65$ \\
\hline 177 & B. melitensis & 180 & 78 & 240 & 460 & $40-45$ \\
\hline V & B. abortus & 180 & 37 & 240 & 480 & $25-30$ \\
\hline
\end{tabular}

Phage reproduction was studied on $B$. abortus 141; Tb phage alone was grown was on both, $B$. abortus S19 (indicated by Tb_19) and 141 (Tb_141) strains.

DNA isolation, genome sequencing and assembly DNA isolation and sequencing

Phage DNAs were isolated by standard procedure by phenol/chloroform extraction. Genomic libraries were quantified using the Qbit (Qbit 2.0 Fluorometer, Life technologies), sheared using the Covaris M220 focused ultrasonicator (Covaris, Inc.), and fragmented QC was performed on the BioAnalyzer 2100 (Agilent Technologies, Inc.). Libraries were subsequently modified using the Illumina Paired End Sample Prep Kit and sequenced on the Illumina MiSeq platform (Illumina, Inc.) per manufacturer's instructions. Raw fastq files for each phage sample were exported and subjected to de novo assembly using CLCBio software (CLC Bio, Aarhus, Denmark) using kmer size $\mathrm{N}=51$, minimum contig size $=1000$. SNP analysis, genome alignments, and Neighbor-Joining [10] dendrograms were generated using Geneious software version 6.1.5 (11). SNP loci exhibiting within-sample heterogeneity in variant calls are illustrated in Table 2 with residues comprising fractions of $<50 \%$ of Illumina read populations designated in brackets. Sanger sequencing was also performed to validate SNP loci. Sequencing primers were designed from the GenBank $\mathrm{Tb}_{M}$ phage reference sequence genome for DNA sequencing on the ABI $3130 \times \mathrm{L}$ sequencer (GenBank: NC_019446.1). Cycle Sequencing PCR was carried out using the BigDye Terminator v3.1 Cycle Sequencing Kit. For the cycle sequencing reaction, each primer was diluted to the $3.2 \mathrm{pmol}$ working concentration and 5-20 ng of template DNAs was used. Sequencing PCRs for each DNA sample (with single primer) was prepared in triplicate. Purification of the sequencing PCR products was performed by BigDyeX Terminator purification kit or by ETOH purification. Purified DNA products were analyzed on the ABI $3130 \times \mathrm{L}$ sequencer (Applied Biosystems). Analysis of the sequencing data was determined using the
DNASTAR- Lasergene 9.1 software (Madison, WI; http://www.dnastar.com).

\section{Nucleotide sequences}

Whole genome sequences for all newly phage genomes in this study were deposited in GenBank: 02_19 (KJ133688), 02_141 (KJ133689), 11sa_19 (KJ133690), 11sa_141 (KJ13 3691), 110_19 (KJ133692), 110_141 (KJ133693), 141_19 (KJ133694), 141_141 (KJ133695), 177_19 (KJ133696), 177_141 (KJ133697), 281_19 (KJ133698), 281_141 (KJ133 699), 544_19 (KJ133700), 544_141 (KJ133701), 1066_19 (KJ133702), 1066_141 (KJ133703), Tb_19 (KJ133704), Tb_141 (KJ133705), V_19 (KJ133706), V_141 (KJ133707).

In addition, arbitrary designations were used to distinguish the multiple $\mathrm{Tb}$ brucellaphage genomes sequenced by other institutes in addition to the $\mathrm{Tb}$ phage sequenced here. These include TbE, TbW, and TbM for the Tb phages grown on the S19 host strain here (KJ133704) and that by Farlow et al [8] (KC556897) and Flores et al. [7] (JN939331), respectively.

\section{Comparative genomics, phylogenetic structure, and selection}

Whole genome alignment data using Geneious software v5.0 [11]. Unrooted Neighbor-Joining phylogenies were performed based on Jukes-Cantor model following best model fit analysis (JC) determined from Bayesian Information Criterion (BIC) in MEGA 5 [12] with boot-strapping performed using 1000 replicates. No outgroup was included as no such significant representative is currently available for the brucellaphage group. Branch lengths were measured in number of substitutions per site with bootstrap performed using 1000 replicates. The significance of testing for the presence of positive (or adaptive) selection was determined by the two tailed Z-test $(\mathrm{P}<0.05)$ using the Nei and Gojobori method 
[13] implemented in MEGA5. The probability of rejecting the null hypothesis indicates strict-neutrality $(\mathrm{dN}=\mathrm{dS})$ in favor of the alternative hypothesis $(\mathrm{dN}>\mathrm{dS})$. Values of $\mathrm{P}$ less than 0.05 are considered significant at the $5 \%$ level and are highlighted. The test statistic ( $d N-d S$ ) (in the Prob column) is shown in the Stat column. dS and dN are the numbers of synonymous and nonsynonymous substitutions per site, respectively. The variance of the difference was computed using the bootstrap method (1000 replicates). The analysis involved 20 nucleotide sequences. All ambiguous positions were removed for each sequence pair. There were a total of 284 positions in the final dataset.

\section{Results and discussion}

\section{Phage lytic activity and host specificity}

$T$ The $\mathrm{Tb}_{E}$ phage was initially cultivated on the B. abortus 141 (serotype I) strain originally isolated in Russia in 1950 , then subsequently grown on $B$. abortus vaccine strain S19. The phenotypic characteristics of all phages are listed in Table 1 . Tb phage propagated on host strain 141 phage displayed a typical slow growth cycle $(190 \mathrm{mi}-$ nutes), on both host bacterial cells however adsorption time was marginally faster on vaccine strain $B$. abortus S19 (180 minutes), average burst size increased on this strain as well (50-53 PFU/cell). Other phages propagated only on the B. abortus 141 host strain also differed in adsorption times (Table 1). Some phages $(02,544)$ adsorbed on the host within two hours, while other phages displayed longer adsorption (Table 1). Average burst size was comparatively low; the highest phage counts were observed for phage 1066 (110-120 PFU/cell). Infection parameters for other brucellaphages grown on S19 were not assessed here.

\section{Genomic comparison and phylogenetic structure}

We performed whole genome sequencing and de novo assembly of ten brucellaphages before and after host propagation from host strain 141 to strain S19 following 2-3 passages. This study is the first report describing whole genome level polymorphisms following serial propagation of brucellaphages on alternate $B$. abortus hosts. Following propagation on S19 each genome sequence was compared to the pre-passaged genome derived from propagation on strain 141 as well as to the brucellaphage genomes reported previously for $\mathrm{Tb}_{M}$ and $\operatorname{Pr}$ (Table 2). Similar genome sizes (41,149 bp), GC content (48.2\%), and gene synteny were observed across the phages in this study. The $\mathrm{Tb}_{E}$ phage genome and other brucellaphage genome sequences were collinear with the published $\mathrm{Tb}_{M}$ phage genome sequence with complete synteny across all genomes analyzed. All ten phages analyzed here showed limited genomic diversity consistent with the genetic homogeneity observed between Pr and $\mathrm{Tb}_{M}$ and common restriction profiles reported previously across other brucellaphages $[7,14,15]$. We found close concordance in genetic similarity between the $\mathrm{Tb}_{W}$ phage [8] and the $\mathrm{Tb}_{E}$ phage sequenced in our study from the Eliava Institute that further supports the unique genetic character of the $\mathrm{Tb}_{M}$ phage [7] which appears atypical [8]. Whole genome sequencing of additional $\mathrm{Tb}$ phage representatives available from the Gamaleya Institute may provide further insight as to the prevalence of unique subtypes distributed by this source. Variations reported previously [8] that are specific to $\mathrm{Tb}_{M}$ alone include: at 6353 - CAAAT to ACCCG $\left(\mathrm{Tb}_{M}\right)$, 11613 - TAT to ATA $\left(\mathrm{Tb}_{M}\right), 12384$ - ATATG to TATAC $\left(\mathrm{Tb}_{M}\right), 13145$ - ATCCGCTACA to CGAATAGCAC $\left(\mathrm{Tb}_{M}\right)$, 13438 - GCGCGCGTAATTT to ATATATGTAATCC $\left(\mathrm{Tb}_{M}\right)$, and at 17184 an insertion of residues GTAGCG. We also noted a variation unique to the Pr strain at position 34533 that displays the sequence ACCGG compared to CAATT in all other brucellaphages. We also observed partial correspondence in the content of select genes encoding mutations when compared to previously published whole genome data [8]. Genomic changes in $\mathrm{Tb}_{E}$ across propagation from host strain 141 to S19 included SNPs predominantly in ORF 21 and the phage tail collar gene at positions 15576/15578 (ORF 21) and positions $2185 / 22178$ (Table 2). A total of five ORFs in our study (\#8,16,21,23, and 27) were also genetically variable across diverse brucellaphages analyzed previously [8]. In addition, a total of five ORFs (\#7, 18, 43, 51, and 58) variable in previously published data [8] showed no variation among the phages analyzed in our study while seven ORFs (\#12, 17, 20, 25, 26, 39, and 42) showed variability in our data set alone, in part possibly due to unique host-factor requisites unique to $B$. abortus strain 141. Overall, genome alignment comparison revealed high pairwise percent identity across all genomes as well as complete conservation of gene number and orientation. This high synteny and complete collinearity in brucellaphages of diverse origin provides further support for potential absence of recombination in brucellaphages and suggests that they may be highly adapted to potential homogenous requisites of host infection expressed by their genetically similar hosts $[7,16,17]$. This is in contrast to many phage populations that appear to evolve through genetic mosaicism $[18,19]$ due to frequent nonhomologous horizontal gene transfer.

The most pronounced differences previously observed between the $\mathrm{Tb}_{M}$ and $\operatorname{Pr}$ brucellaphages genomes include two major indel regions [7]. The first major indel region encoded by $\mathrm{Tb}$ extends ORF 21 that encodes a structural protein with significant orthology to hypothetical proteins in other diverse phage. The second major indel region extends the tail fiber protein (ORF 28) and 
Table 2 Nucleotide polymorphisms in whole genome sequences of the 22 brucellaphage genomes studied

\begin{tabular}{|c|c|c|c|c|c|c|c|c|c|}
\hline$\overline{{ }^{\mathrm{a}} \mathrm{ORF}}$ & 7 & 8 & 12 & 12 & 13 & 17 & 20 & 21 & \\
\hline
\end{tabular}

\begin{tabular}{llllll}
$\mathbf{2 1}$ & $\mathbf{2 1}$ & $\mathbf{2 1}$ & $\mathbf{2 3}$ & $\mathbf{2 3}$ & $\mathbf{2 3}$ \\
\hline 16003 & 16012 & 16064 & 16700 & 16952 & 16984
\end{tabular}

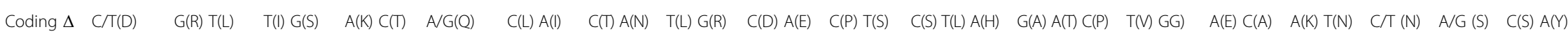

Codon p. 3rd 2nd 2nd 2nd 1st 1st 2nd 1st 3rd 1st 2nd 2nd 1st 2nd 2nd

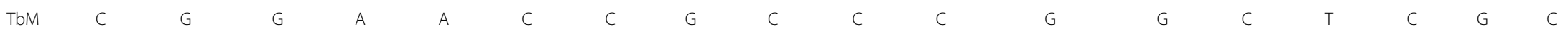

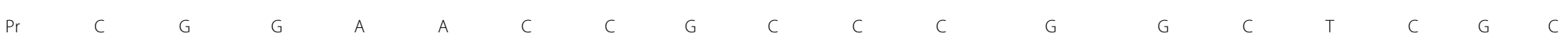

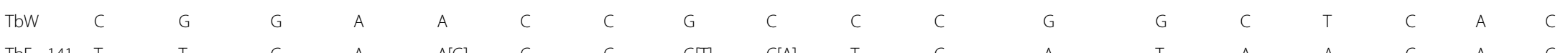

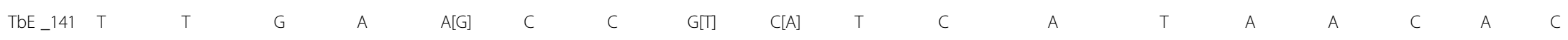

TbE _19 T

02_141 T

02_19

11sa_141 T

11sa_19 T

141_141 T

141_19 T

177_141 T

77_19

281_141 T

281 19 T

544_141 T

544_19 T

1066_141 T

1066_19 T

V_141

V_19
110_141

110_19 T

ORF 25

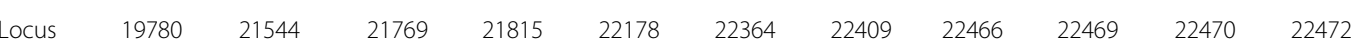

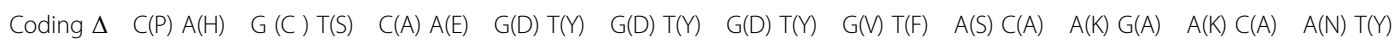


Table 2 Nucleotide polymorphisms in whole genome sequences of the 22 brucellaphage genomes studied (Continued)

\begin{tabular}{|c|c|c|c|c|c|c|c|c|c|c|c|c|c|c|c|c|c|}
\hline TbE_141 & C & $\bar{G}$ & C & $\mathrm{G}[\mathrm{T}$ & $\bar{T}$ & $\bar{T}$ & $T$ & $A$ & $\bar{G}$ & C & $\bar{T}$ & $\bar{G}$ & $\bar{C}$ & C & $\bar{C}$ & $\bar{T}$ & $\bar{T}$ \\
\hline TbE_19 & $C[A]$ & G & c & G & $\mathrm{T}[\mathrm{G}]$ & T & $\mathrm{T}$ & A & G & C & T & G & C & C & C & $\mathrm{T}$ & T \\
\hline 02_141 & C & G & C & G & G & T & G & A & G & C & T & $\mathrm{GT}$ & C & C & C & T & T \\
\hline 02_19 & C & G & C & G & G & T & G & A & G & C & $\mathrm{T}$ & $\mathrm{G}(\mathrm{T}$ & C & C & C & $\mathrm{T}$ & $\mathrm{T}$ \\
\hline 11sa_141 & C & G & C & G & G & $\mathrm{G}[\mathrm{T}$ & G & A & A & A & T & G & T & C & C & $\mathrm{T}$ & $\mathrm{T}$ \\
\hline 11sa_19 & c & G & C & G & G & T & G & A & G & C & T & G[T] & C & C & C & $\mathrm{T}$ & $\mathrm{T}$ \\
\hline 141_141 & C & G & $C[A]$ & G & G & T & G & A & A & A & T & G & C & C & C & $\mathrm{T}$ & $\mathrm{T}$ \\
\hline 141_19 & C & G & $C[A]$ & G & G & T & G & A & A & A & T & G & C & C & C & T & $\mathrm{T}$ \\
\hline 177_141 & $C[A]$ & G & C & G & T & $\mathrm{T}$ & $T$ & A & G & C & $\mathrm{T}$ & G & C & C & C & $\mathrm{T}$ & $\mathrm{T}$ \\
\hline 177_19 & $C[A]$ & G & C & G & T & T & $T$ & A & G & C & $\mathrm{T}$ & G & C & C & C & $\mathrm{T}$ & $\mathrm{T}$ \\
\hline 281_141 & $C[A]$ & G & C & G & T & T & $T$ & A & G & C & T & G & C & C & C & $\mathrm{T}$ & $\mathrm{T}$ \\
\hline 281_19 & c & T & $C[A]$ & G & G & $\mathrm{G}[\mathrm{T}$ & G & A & A & A & $T$ & G & C & C & C & $T$ & $\mathrm{~T}$ \\
\hline 544_141 & c & G & c & G & G & $\mathrm{T}$ & $T$ & A & $A[G]$ & $\mathrm{A}[\mathrm{C}]$ & T & G & $\mathrm{T}[\mathrm{C}]$ & C & C & $\mathrm{T}$ & $\mathrm{T}$ \\
\hline 544_19 & c & G & C & G & G & T & $T$ & A & $\mathrm{A}[\mathrm{G}]$ & $\mathrm{A}[\mathrm{C}]$ & T & G & $\mathrm{T}[\mathrm{C}]$ & C & c & $\mathrm{T}$ & $\mathrm{T}$ \\
\hline 1066_141 & C & G & $C[A]$ & G & G & $\mathrm{G}[\mathrm{T}$ & G & A & A & A & $T$ & G & c & c & c & $\mathrm{T}$ & $\mathrm{T}$ \\
\hline 1066_19 & $C[A]$ & G & $C[A]$ & G & $\mathrm{G}[\mathrm{T}$ & $\mathrm{G}[\mathrm{T}$ & $\mathrm{G}[\mathrm{T}$ & A & $\mathrm{A}[\mathrm{G}]$ & $\mathrm{A}[\mathrm{C}]$ & $T$ & $\mathrm{G}[\mathrm{T}$ & $\mathrm{T}[\mathrm{C}]$ & C & c & $\mathrm{T}$ & $\mathrm{T}$ \\
\hline V_141 & c & G & c & G & G & $\mathrm{G}[\mathrm{T}$ & $T$ & A & A & A & T & G & T & C & c & $\mathrm{T}$ & $\mathrm{T}$ \\
\hline V_19 & c & G & C & G & T & T & G & A & G & c & T & $\mathrm{G}(\mathrm{T}]$ & c & C & c & $\mathrm{T}$ & $\mathrm{T}$ \\
\hline 110_141 & C & G & C & G & G & T & G & A & G & C & $T$ & T & C & C & c & $\mathrm{T}$ & $\mathrm{T}$ \\
\hline 110_19 & A & G & C & G & T & T & $T$ & A & G & C & T & G & C & C & C & T & $\mathrm{T}$ \\
\hline
\end{tabular}

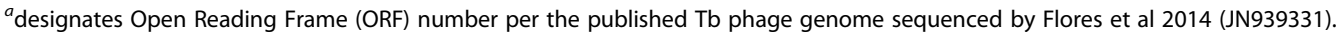


encodes an additional gene (ORF29) that exhibits significant similarity to pectin-lyase-like domain containing carbohydrate binding proteins. We note the majority of variation in our study and that presented in a recent report [8] suggest this genome block encodes most of the genetic variability in brucellaphage genomes. The cellular receptor(s) used by brucellaphages have not been identified though it is possible that variations in distinct requisites of attachment across host organisms may have induced variations in tail fiber length or specificity as well as a requirement for the putative carbohydrate binding protein (ORF29). All phages in this analysis lacked the two major deletions present in the Pr phage lytic for B. melitensis (Figure 1). The gene variations within this region including the carbohydrate binding protein and the tail fiber protein, may reflect adaptations to host range determinants distinguishing phage lytic for B. abortus or B. melitensis, however such speculation must be experimentally confirmed. Genetic co-variations observed here shared among multiple phages lytic for B. abortus may represent more fine-scale adaptations occurring from propagation on a single host species, though at present this remains speculative. Phage 11sa showed the greatest variation across propagation on S19 and 141 (Figure 2). Phages 02, 141, and 177 exhibited the greatest genetic stability and showed no difference in topological placement following propagation on strain 19 while only a moderate change in topological placement was noted for phage 544 (Figure 2). While isolation of brucellaphages lytic for their host of origin is rare, resistant sub-clones have been isolated from field samples and laboratory stocks [20,21]. Previous studies on the 544 host strain reported isolation of a phage resistant descendent strain (544-FS) refractory to infection (though not adsorption) by $\mathrm{Tb}, \mathrm{S} 708$ and other brucellaphages [20,21]. Resistant host strains giving rise to phage lytic for their smooth variants is a possibly explanation though this has not be reported.

\section{Candidate loci for host specificity}

We noted a total of 24 polymorphic loci within 13 genes including two SNPs within the 3' non-coding terminal region (Table 2, Figure 1) within the core set of Georgian brucellaphages propagated in this study. For comparison we included 11 additional loci reported previously that do not share identity with the Georgian phages analyzed here (Table 2). We noted a total of two synonomous mutations and 22 non-synonomous mutations (Table 3) within our core set of phages. Only phage 02 showed no polymorphisms on propagation from strain 141 to strain 19 . Genomic changes predominantly occurred at identical sites across separate phage lineages including withinsample heterogeneity. Mixed within-sample genotypes (two-states) were observed across all loci in at least a single phage. We speculate such loci indicate emergence or maintenance of particular residues imparting a fitness cost or benefit. Though it is possible sequencing error could play some role in this variation across Illumina sequence read populations we note that in all observed sites one or both of the two alternate residues are observed in up to $100 \%$ of reads at the same individual locus in multiple alternate phages across alternate Illumina library preparations. Whether such loci indicate the potential presence of less-fit minority variants, defective virions carried over during the brucellaphage life cycle or emergence of actual quasispecies clone subsets with variable fitness must await experimental validation. In addition, several SNP loci were also observed that altered restriction site modification signatures (Table 4). Genes containing the greatest number of shared and unique polymorphisms

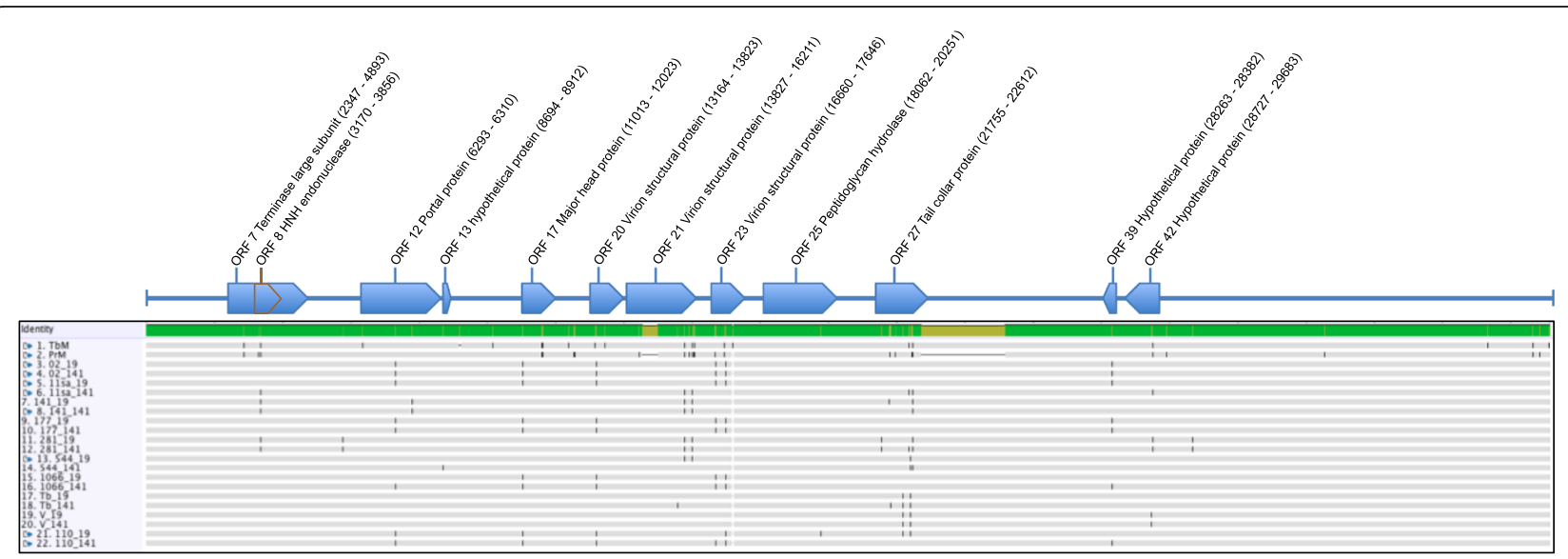

Figure 1 Whole genome alignments of all genomes analyzed in this study. SNPs are indicated by vertical black lines, phages are designated by phage ID number followed by underscore and propagating host strain name. Open Reading Frames number and location are illustrated above the alignment. 


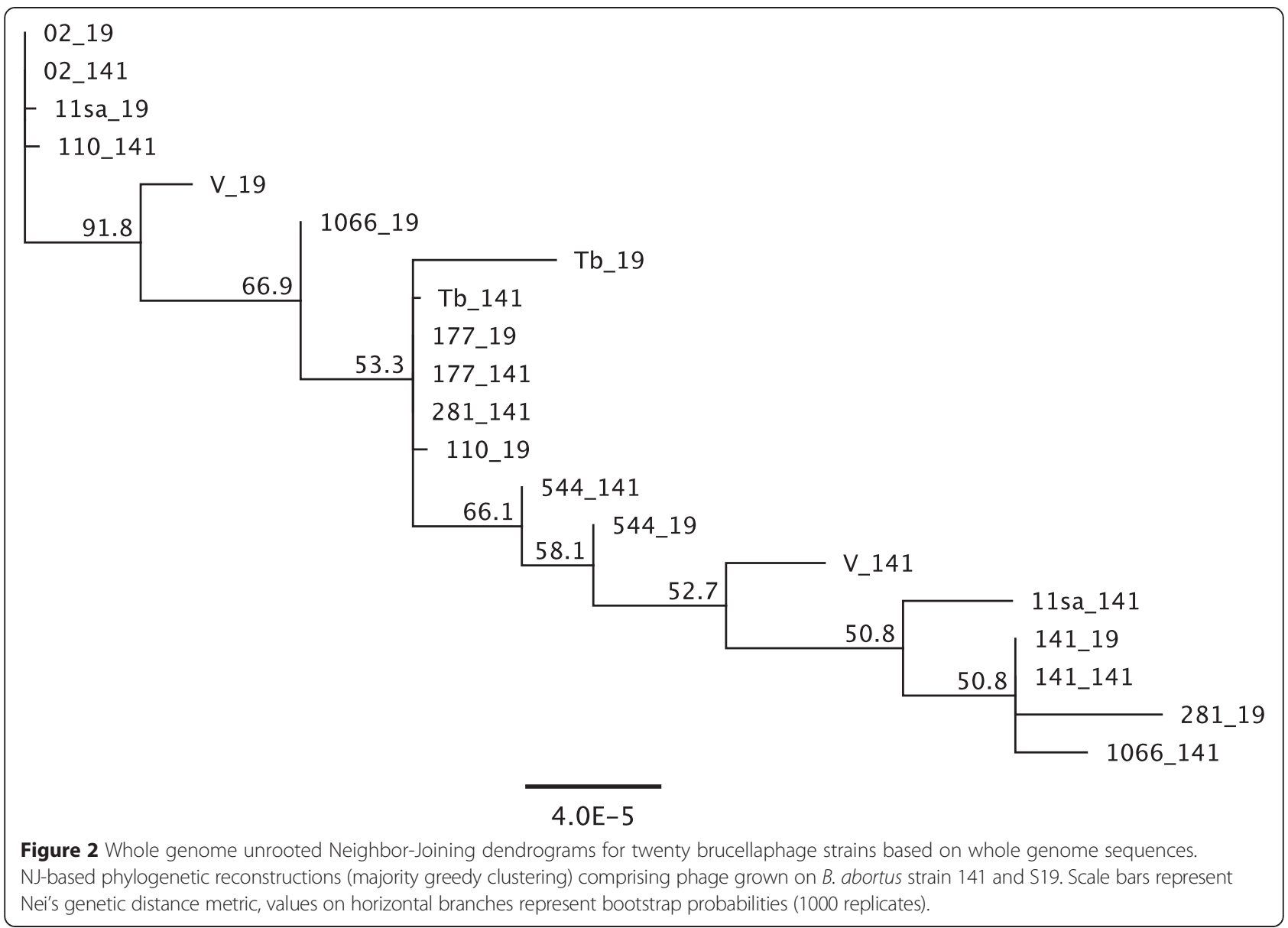

Table 3 Annotated list of open reading frames showing major variations in synonomous and non-synonomous polymorphisms

\begin{tabular}{|c|c|c|c|c|c|c|c|c|}
\hline Annotation & ORF\# & Start & End & Length & Direction & Coding $\Delta$ & \# Loci $^{b}$ & Passage $^{c}$ \\
\hline Putative HNH endonuclease & 8 & 3,170 & 3,856 & 687 & forward & $\mathrm{S}$ & 1 & $\mathrm{~N}$ \\
\hline Putative portal protein & 12 & 6,370 & 8,694 & 2,325 & forward & $\mathrm{N}$ & 2 & Y \\
\hline Hypothetical protein & 13 & 8,694 & 8,912 & 219 & forward & S & 1 & Y \\
\hline Structural protein & 16 & 9,822 & 11,003 & 1,182 & forward & N & 1 & N \\
\hline Major head protein & 17 & 11,013 & 12,023 & 1,011 & forward & N & 1 & Y \\
\hline Structural protein & 20 & 13,164 & 13,823 & 660 & forward & N & 1 & Y \\
\hline Structural protein (neck motif) & 21 & 13,827 & 16,211 & 2,385 & forward & N & 5 & $\mathrm{Y} / \mathrm{N}$ \\
\hline Structural protein (peptidase_S74) & 23 & 16,660 & 17,646 & 987 & forward & $\mathrm{N}$ & 2 & $\mathrm{Y} / \mathrm{N}$ \\
\hline Putative peptidoglycan hydrolase & 25 & 18,062 & 20,251 & 2189 & forward & $\mathrm{N}$ & 1 & Y \\
\hline Structural protein & 26 & 20,251 & 21,753 & 1,503 & forward & $N$ & 1 & Y \\
\hline Putative tail collar protein & 27 & 21,755 & 22,612 & 858 & forward & S-N & 7 & $\mathrm{Y} / \mathrm{N}$ \\
\hline Hypothetical protein & 39 & 28,262 & 28,382 & 121 & reverse & $N$ & 1 & Y \\
\hline Hypothetical protein & 42 & 28,727 & 29,683 & 957 & reverse & S-N & 2 & Y \\
\hline
\end{tabular}

artholog encoded by Brucella 83/13.

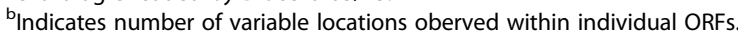

${ }^{\mathrm{c}}$ Indicates loci showing polymorphism among passaged strains $(\mathrm{Y})$ versus variants present only within the $\operatorname{TbM}$ and $\operatorname{Pr}$ reference strains $(\mathrm{N})$. 
Table 4 SNP loci resulting in restriction site modifications

\begin{tabular}{|c|c|c|c|c|c|c|c|c|c|c|c|c|c|c|c|c|}
\hline \multicolumn{17}{|c|}{ Restriction sites } \\
\hline & Haell & Hhal & Cfol & Alul & Hinfl & Msel & Haelll & Taql & Sall & Hincll & Accl & Mspl & Hpall & Mbol & Sau3AI & Ndel \\
\hline 3357 & G & G & G & - & - & - & - & - & - & - & - & - & - & - & - & - \\
\hline 11046 & - & - & - & a & A & & - & - & - & - & - & - & - & - & - & - \\
\hline 13192 & - & - & - & - & - & A & - & - & - & - & - & - & - & - & - & - \\
\hline $15576-8$ & - & - & - & - & - & - & - & $\mathrm{t}$ & $t$ & $t$ & $\mathrm{t}$ & - & - & - & - & - \\
\hline 15774 & - & - & - & - & - & - & C & - & - & - & - & - & - & - & - & - \\
\hline 15775 & - & - & - & - & - & $\mathrm{T}$ & - & - & - & - & - & - & - & - & - & - \\
\hline 15996 & - & G & G & - & - & - & - & - & - & - & - & $C$ & $C$ & - & - & - \\
\hline 19780 & - & - & - & - & - & - & $a$ & - & - & - & - & - & - & - & - & - \\
\hline 22178 & - & - & - & - & - & - & - & - & - & - & - & - & - & t & t & - \\
\hline 22470 & - & - & - & - & - & - & - & - & - & - & - & - & - & - & - & $a$ \\
\hline 28322 & - & - & - & - & - & - & $\mathrm{t}$ & - & - & - & - & - & - & - & - & - \\
\hline
\end{tabular}

Note: SNPs in upper case letters designate generation of restriction sites, lower case letters designate abolished restriction sites.

included putative genes encoding the tail collar protein and the structural protein encoded by ORF 21 (putative neck region). Two SNP loci at positions 22364 and 22470 within the tail collar protein gene (ORF 27) in our data also displayed variability across $\mathrm{Tb}\left(\mathrm{Tb}_{W}\right)$, Firenze (Fz), Weybridge (Wb), S708, Berkeley (Bk) and R/C brucellaphages as reported previously [8]. Similar to previous data [8], the gene encoding the putative phage tail collar protein showed the most pronounced level of variation with nine coding changes comprising 27\% of total SNPs among the phages analyzed. Along with the ORF 21 (structural protein), this gene is the most hyper-variable region in the genome across the brucellaphages analyzed here (Tables 2 and 3, Figure 1) and is consistent with that observed previously in other brucellaphages [8]. Three of the observed mutations in the collar protein gene resulted in stop codons and may represent premature termination of the encoded protein product. Previous speculation [8] suggested coding changes in the tail collar protein may alter the conformation/activity of the attached tail fiber protein through steric alterations or perhaps affect conformational interactions with the portal protein mediating viral DNA injection. Phage tail collar proteins assemble into ordered arrays and play a central role in binding the phage portal protein and orienting phage tail fibers [22]. Though speculative, this protein may be positioned at the nexus of both phage attachment (binding tail fiber protein) and DNA injection (phage portal protein) and could be subjected to enhanced selective forces imparted by strain-specific variations in functional requisites. For example, changes in the efficiency of genome injection (portal protein) across phages or absorption capacity (tail fibers) could select for structural modifications effecting collar organization, folding, or stability. Future experimental studies are needed to evaluate these speculations. To test whether the variability observed in the tail collar protein may be under selective pressure, we tested for the presence of positive selection acting on the collar protein gene and ORF 21 using a codon-based test of positive selection averaging over all sequence pairs. Application of the Bayesian Information Criterion (BIC) identified the Jukes-Cantor model as the best fit of the observed substitution pattern. Results were significant for positive selection acting in the tail collar gene with an overall $\mathrm{dN}-\mathrm{dS}=2.10(\mathrm{P}=0.018)$. In contrast, positive selection does not appear to be acting on ORF $21 \mathrm{dN}-\mathrm{dS}=1.48(\mathrm{P}=0.071)$.

We also observed common mutations within the gene encoding the putative brucellaphage $\mathrm{HNH}$ endonuclease (ORF 8, R63L) (Table 2, Figure 1). HNH endonucleases are homing endonucleases utilized in DNA binding, nicking and degradation, of viral and bacterial nucleic acids. The HNH catalytic domain of phage and bacterial homing endonucleases consist of the conserved catalytic residues His-Asn-His as well as a zinc-binding site $[\mathrm{CxxC}]$ and a zinc ribbon (ZR) domain with potentially multiple zincbinding sites ([CxxxxC], [CxxxxH], [CxxxC], [HxxxH], $[\mathrm{CxxC}]$ or $[\mathrm{CxxH}])$ present at the $\mathrm{N}$-terminus or $\mathrm{C}$ terminus [23]. These proteins require divalent transition metals for endonuclease activity and variations in zinc binding mediate differences in enzymatic activity. In brucellaphage, it is possible this protein functions as a replication initiation protein nicking phage dsDNA during genome replication. In our analysis, phages $\mathrm{Tb}_{M}, \mathrm{Pr}$, 11sa-141, 141-141, 141-19, and 281-19 possess an arginine at coding position 63 while all other phage including $\mathrm{Tb}$ encode a leucine at this position. The location of the active site within the brucellaphage $\mathrm{HNH}$ endonuclease protein lies between residues 160 to 184 within the ZR domain and is flanked by two potential zinc-binding sites (data not shown). The variable site R63L is located within this domain flanked by multiple potential zinc binding sites within the ZR domain. Furthermore, in contrast to 
the flanking position of genes encoding terminase subunits and $\mathrm{HNH}$ endonucleases (within $5 \mathrm{ORFs}$ ) previously observed in tailed Kala et al [24], we note that the nontailed brucellaphages, encode the $\mathrm{HNH}$ endonuclease gene overlapping the mid-portion of the large subunit terminase gene. This finding further supports a potential functional association between these proteins as well as both the conservation of terminase associated $\mathrm{HNH}$ proteins and existence of a potentially distinct functional subfamily of the $\mathrm{HNH}$ endonucleases noted previously by Kala et al [25]. The observed SNP at position 3357 that results in a putative R63L mutation in the $\mathrm{HNH}$ endonuclease is silent in the putative large terminase subunit protein. The putative intein-encoded $\mathrm{HNH}$ endonuclease gene located out of frame within the large terminase subunit is present in all annotated brucellaphage genomes published to date $(7,8)$. Inteins are parasitic genetic elements that often encode $\mathrm{HNH}$ endonucleases to confer mobility. Sequence variation within these genes across brucellaphage host range variants have been previously noted $(7,8)$. In our analysis, sequence similarity searches did not reveal signatures of other parasitic genetic elements in this region, such as group I and Group II introns. We speculate that the sequence variation in the inteinencoded $\mathrm{HNH}$ endonuclease gene and the terminase may represent stochastic variation, or, result from fine-scale differences in the biological properties of these proteins during phage replication within the bacterial host milieu. Such factors may include parameters effecting the evolutionary maintenance of the intein- $\mathrm{HNH}$ parasitic element such as intein homing efficiency and target nucleotide binding, DNA cleavage and splicing, or co-factor binding specificity. In any case, future experimental investigation may identify the role, if any, of the observed sequence variation.

Comparative sequence analysis also revealed a sequence comprising 299 bp residues (26701-27600; $\mathrm{Tb}_{M}$ ) spanning two hypothetical proteins that showed significant similarity to a region directly upstream of a phage integrase motif in the genome of Ochrobactrum anthropi. This orthologous sequence shared between brucellaphage and $O$. anthropi, is interesting as $O$. anthropi is the closest known relative to the Brucellaceae $[25,26]$. It is postulated that major clades in the Brucellaceae family that now maintain an intracellular lifestyle evolved from Ochrobactrum-like relatives free living in soil and plant-associated rhizosphera. The phagerelated Ochrobactrum-like motif observed in the genome of brucellaphages may reflect common ancestry between phages infecting these distantly related bacterial hosts though with low evolutionary signal due to the likely divergent selective pressures separating their current niches. In addition, a 36 bp residue sequence at the $3^{\prime}$ end of this region (27218-27253) displayed significant similarity to a prophage antirepressor encoded by $B$. canis strain HSK
A52141. This sequence is also shared broadly across other Brucella host species. Further BLAST analysis also revealed a motif comprising 27 nucleotide residues at the 3 ' terminus of all phages showed significant similarity to a single CRISPR spacer sequence in the CRISPR database (http://crispr.u-psud.fr/crispr/) encoded by Staphylothermus marinus F1. This orthologous sequence shared between brucellaphage genomes and the $S$. marinus CRISPR spacer showed no significant similarity to other available sequences in GenBank. Interestingly, phage resistance systems such as CRISPRs are notably absent in Brucella genomes with the exception of questionable CRISPR-like genetic structures in select $B$. melitensis and $B$. pinnipedialis strains (http://crispr.u-psud.fr/crispr/).

\section{Conclusions}

We surveyed whole genome level diversity in phage lytic for $B$. abortus and revealed fine-scale patterns in the genetic structure of these phages as they are propagated on vaccine strains within this species. Previous studies showed the relatively homogenous genomes of brucellaphages derived from diverse Brucella species express frequent polymorphisms in the phage collar protein that may reflect host selection. Our data extend these results confirming such diversity on a finer scale during propagation within the $B$. abortus species and illustrate multiple common sequence variations across similar genes that frequently display within-sample genetic heterogeneity. These data also provide a comparison of candidate loci for potential genotype-phenotype associations that can be explored in the Tb phage model while also yielding additional genomes for future reference in comparative studies involving the molecular evolution of brucellaphages.

\section{Competing interests}

The authors declare that they have no competing interests.

\section{Authors' contributions}

$E T, N S, I A, S G, N B$, and LK performed phage isolation, propagation, and DNA extractions. AK, ET, and NS performed the genome sequencing. MK and JF conceived of the study design. JF and MK drafted the manuscript. JF performed the sequence analysis. All authors read, reviewed and approved the final manuscript.

\section{Acknowledgments}

The work was made possible by support provided by the US Defense Threat Reduction Agency through the Cooperative Biological Engagement Program in Georgia (DTRA TAP-3 Project). We would like to thank Cheryl Gleasner for her technical support on Illumina sample processing procedures.

\section{Author details}

${ }^{1}$ George Eliava Institute for Bacteriophages, Microbiology and Virology, Tbilisi, Georgia. 'Lugar Center for Public Health Research at National Center for Disease Control, Tbilisi, Georgia. ${ }^{3}$ Academic Engagement Program (AEP) Pennsylvania State University, University Park, State College, USA. ${ }^{4}$ Farlow Scientific Consulting Company, LLC, Lewiston, Utah, USA.

Received: 1 August 2014 Accepted: 24 March 2015

Published online: 22 April 2015 
References

1. Dean AS, Crump L, Greter H, Schelling E, Zinsstag J. Global burden of human brucellosis: a systematic review of disease frequency. PLoSNegl Trop Dis. 2012;6:e1865.

2. Rosenberg E, Stackebrandt E, Thompson F, Lory S, DeLong E. The Prokaryotes A Handbook on the Biology of Bacteria. Springer-Verlag New York, LLC 2006, Vol. 5: Proteobacteria: Alpha and Beta Subclasses. 3rd Edition.

3. Corbel MJ, Thomas EL. The Brucella-Phages: Their Properties, Characterization and Applications. In: Booklet 2266, Ministry of Agriculture, Fisheries and Food, Pinner, Middlesex, UK. 1980

4. Jones $L M$, Merz GS, Wilson JB. Phage typing reactions on brucella species. Appl Microbiol. 1968;16(8):1179-90.

5. McDuff CR, Jones LM, Wilson JB. Characteristics of brucellaphage. J Bacteriol. 1962;83(2):324-9.

6. Stinebring WR, Braun W. Brucellaphage. J Bacteriol. 1959;78(5):736-7.

7. Flores V, López-Merino A, Mendoza-Hernandez G, Guarneros G. Comparative genomic analysis of two brucellaphages of distant origins. Genomics. 2012;99(4):233-40

8. Farlow J, Filippov AA, Serqueev KV, Hang J, and MP Nikolich. Comparative whole genome analysis of six diagnostic brucellaphages. Gene. 2014. doi:10.1016/j.gene.2014.01.018.

9. Adams MH. Bacteriophages. New York: Interscience; 1959.

10. Saitou N, Nei M. The neighbor-joining method: a new method for reconstructing phylogenetic trees. Mol Biol Evol. 1987:4:406-25.

11. Drummond A, Kearse M, Heled J, Moir R, Thierer T, Ashton B, et al. Geneious v2.5. 2006. Available from http: //www.geneious.com/

12. Tamura K, Peterson D, Peterson N, Stecher G, Nei M, Kumar S. MEGA5: Molecular evolutionary genetics analysis using maximum likelihood, evolutionary distance, and maximum parsimony methods. Mol Biol Evol. 2011;28:2731-9.

13. Nei M, Gojobori T. Simple methods for estimating the numbers of synonymous and nonsynonymous nucleotide substitutions. Mol Biol Evol. 1968:3:418-26.

14. Segondy M, Allardet-Servent A, Caravano R, Ramuz M. Common physical map of four Brucella bacteriophage genomes. FEMS Microbiol Lett. 1988;56(2):177-82.

15. Zhu CZ, Xiong HY, Han J, Cui BY, Piao DR, Li YF, et al. Molecular characterization of Tb, a new approach for an ancient brucellaphage. Int J Mol Sci. 2009;10(7):2999-3011.

16. Verger JM, Grimont F, Grimont PAD, Grayon M. Brucella, a monospecific genus as shown by deoxyribonucleic acid hybridization. Int J Syst Bacteriol. 1985;35:292-5.

17. Verger JM, Grimont F, Grimont PAD, Grayon M. Taxonomy of the genus Brucella. Ann Inst Pasteur Microbiol. 1987;1987(138):235-8.

18. Casjens S, Hendrix R. Bacteriophages and the bacterial genome. In: Higgins NP, editor. The Bacterial Chromosome. Washington DC: ASM Press; 2005.

19. Hendrix RW, Smith MC, Burns RN, Ford ME, Hatfull GF. Evolutionary relationships among diverse bacteriophages and prophages: all the world's a phage. Proc Natl Acad Sci USA. 1999;96:2192-7.

20. Corbel MJ, Morris JA. Studies on a smooth phage resistant variant of Brucella abortus II. Mechanism of phage resistance. Br J Exp Pathol. 1975;56(1):1-7.

21. Harrington Jr R, Bond DR, Brown GM. Smooth phage-resistant Brucellaabortus from bovine tissue. J Clin Microbiol. 1977;5(6):663-4.

22. Kurtboke, Ipek. Bacteriophages. InTech, Rijeka, Croatia, 2012, ISBN 978-953-51-0272-4

23. Xu SY, Gupta YK. Natural zinc ribbon HNH endonucleases and engineered zinc finger nicking endonuclease. Nucleic Acids Res. 2013;41(1):378-90.

24. Kala S, Cumby N, Sadowski PD, Hyder BZ, Kanelis V, Davidson AR, et al. HNH proteins are a widespread component of phage DNA packaging machines. Proc Natl Acad Sci. 2014;11(16):6022-7.

25. Velasco J, Romero C, Lopez-Goni I, Leiva J, Diaz R, Moriyon I. Evaluation of the relatedness of Brucella spp. and Ochrobactrumanthropiand description of Ochrobactrumintermedium sp. nov., a new species. Int I Syst Bacteriol. 1998;48:759-68.

26. Yanagi M, Yamasato K. Phylogenetic analysis of the family Rhizobiaceae and related bacteria by sequencing of 165 rRNA gene using PCR and DNA sequencer. FEMS Micro Lett. 1993;107(1):115-20.

\section{Submit your next manuscript to BioMed Central and take full advantage of:}

- Convenient online submission

- Thorough peer review

- No space constraints or color figure charges

- Immediate publication on acceptance

- Inclusion in PubMed, CAS, Scopus and Google Scholar

- Research which is freely available for redistribution 